\title{
Preliminary results of spat collection of Rayed pearl oyster (Pinctada radiata) in Turkey
}

\section{Işınlı inci istiridyesi (Pinctada radiata) spatlarının toplanması üzerine Türkiye'deki ilk sonuçlar}

\section{Selçuk Yiğitkurt* (D) • Aynur Lök • Ali Kırtık • Aysun Küçükdermenci • Evrim Kurtay}

Faculty of Fisheries, Ege University, 35100 Bornova-izmir, Turkey

Corresponding author: selcuk.yigitkurt@ege.edu.tr

\section{How to cite this paper:}

Yiğitkurt, S., Lök, A., Kırtık, A. Küçükdermenci, A. \& Kutay, E. (2017). Preliminary results of spat collection of Rayed pearl oyster (Pinctada radiata) in Turkey. Ege Journal of Fisheries and Aquatic Sciences, 34(2): 211-218. doi:10.12714/egejfas.2017.34.2.13

\begin{abstract}
This research was carried out in Karantina Island to find pearl oyster (Pinctada radiata Leach, 1814) spat collection efficiency of bag and shell collector types. Environmental limits such as temperature, salinity, chlorophyll-a, and total particulate matter (TPM) were monitored during the study period. The abundance of P.radiata spat were found on the bag collector as $11.66 \pm 2.08$ ind. $/ 0.91 \mathrm{~m}^{2}$ at the first time in August. At the same month the highest temperature and salinity were recorded as $27^{\circ} \mathrm{C}$ and $36.5 \%$, respectively. G2 (group 2/deployed on June 25) performed the highest spat collection for the bag collectors while G4 (deployed on August 29) for the shell collector was more successful during the research. A total of $172 \pm 34.39$ P. radiata spat were collected during the study. $119 \pm 22.60$ $(69.18 \%)$ individuals (ind) were harvested from bag collector and $53 \pm 11.78(30.82 \%)$ from shell collector. While the avarege abundance of $P$. radiata spat were 7.26 ind. $/ \mathrm{m}^{2}$ on bag collectors, it was 3.23 ind. $/ \mathrm{m}^{2}$ on the shell collectors. A significant difference was determined in the total abundance of $P$. radiata between the collector groups $(p<0.05)$. The length range of Pinctada spat on the bag collectors was between $4.47-33.9 \mathrm{~mm}$ while it was $4.66-18 \mathrm{~mm}$ on the shell collectors. Fouling organisms (barnacles, polychaete, bryozoa) and predators (crabs, gastropods) of pearl oyster were also found on the collector groups. Results of the study established that bag collectors were more effective to collect the pearl oyster spat than the shell collectors.
\end{abstract}

Keywords: Pinctada radiata, rayed pearl oyster, spat collector, Karantina Island

Öz: Bu çalışma torba ve kabuk kolektör tipleriyle ıșınlı inci istiridyesi (Pinctada radiata, Leach 1814) spatlarının toplama verimliliğini bulmak için Karantina Adası'nda gerçekleştirildi. Kolektörler altı grup olarak hazırlandı ve uzun halat sistemi üzerine yerleştirildi. Sıcaklık, tuzluluk, klorofil-a ve toplam partikül madde miktarı gibi çevresel parametreler çalışma periyodu boyunca izlendi. $P$. radiata spatı ilk olarak Ağustos ayında torba kolektörlerde 11,66 $\pm 2,08$ birey/0,91 $\mathrm{m}^{2}$, kabuk kolektörlerde ise $2,33 \pm 1,52$ birey $/ 0,91 \mathrm{~m}^{2}$ olarak tespit edildi. Aynı ayda en yüksek sıcaklık ve tuzluluk değerleri sırasıyla $27^{\circ} \mathrm{C}$ ve $\% 036,5$ olarak kayıt edildi. Çalışma boyunca, torba kolektörlerde en iyi spat toplama performansını Grup 2 (G2-25 Haziran'da yerleştirildi) gösterirken, kabuk kolektörde G4 (29 Ağustos'ta yerlestirildi) daha başarılı olmuştur. Toplamda $172 \pm 34,39$ spat toplandı. $119 \pm 22,60(69.18 \%)$ spat torba kolektörlerle toplanırken, $53 \pm 11.78(30.82 \%)$ spat kabuk kolektörlerle toplandı. Ortlama 7,26 birey $/ \mathrm{m}^{2}$ Pinctada spatı torba kolektörlerde, 3,23 birey $/ \mathrm{m}^{2}$ kabuk kolektörde belirlenmiştir. Kolektör grupları arasında toplam spat bulunma farkının önemli derecede anlamlı çıktığı görülmüştür $(p<0,05)$. Torba kolektörler üzerinde bulunan Pinctada spatlarının boy aralığı 4,47-33,9 mm arasında değişirken, kabuk kolektörler üzerinde bulunan spatların boy aralığı 4,66-18 mm arasında değişim gösterdi. Fouling organizmalar (Balanus, Poliket, Bryozoa) ve predatör türler de (yengeç ve gastropod) kolektör gruplarında bulundu. Çalışma sonucunda torba kolektörlerin kabuk kolektörlere oranla daha verimli olduğu belirlenmiştir.

Anahtar kelimeler: Pinctada radiata, Işınıı Inci Istiridyesi, spat toplama, Karantina Adası

\section{INTRODUCTION}

Pinctada radiata (Leach, 1814), known as the rayed pearl oyster, is distributed of the north-western Indian Ocean and the Red Sea (Al-saadi, 2013), Mediterranean (Göksu et al., 2002) and Aegean coasts (Yiğitkurt, 2011). Culturing pearl oyster can supply to economic and social benefits and its techniques does not depend on using big capital (Lodeiros et al., 2002). Pearl culture facilities create employee and correspondingly a business outcome because of its marine protein and shells with nacre. By these features it comes to be proper species for investment (Baqueiro and Castagna, 1988; Gervis and Sims, 1992).

Pearl production consists of four principal stages; spat collection or larval production, growth, nucleus implant, and harvesting (Chellam et al., 1991). Spat collection is the first stage of bivalve culture (Beer and Southgate, 2000). This step is economical in comparision to larval culture procedures; hence; various types of collector materials are being used for a 
long time (Friedman and Bell, 1996). Collector materials should be long-suffering, cheap, and easy to access besides ideal substrate for bivalve spat (Vakily, 1989). Various materials were used for spat collection of pearl oyster. Some of those are cedar springs, mollusk shells, old fishing nets for $P$. martensii in Japan (Alagarswami, 1991); oyster baskets, nylon mesh, nylon frills for $P$. fucata ( $=P$. radiata, $P$. imbricata) in India (Nayar et al., 1978; Victor et al., 1987); neutron tube, shade mesh, Pemphis acidula sprigs for $P$. margaritifera in French Polynesia (Coeroli et al., 1983); wooden boards, split bamboo for P. margaritifera in Sudan (Rahma and Newkirk, 1987); nylon gill net in mesh bags for $P$. mazatlanica and Pteria sterna in Mexico (Monteforte and Garcia-Gasca, 1994); shade mesh sheet/strips and plastic sheet/strips for $P$. margaritifera in Solomon Islands (Friedman et al. 1998); shade cloths in mesh bags for Pinctada spp. and Pteria spp. in Australia (Beer and Southgate, 2000); onion bags for $P$. imbricata (Urban, 2000).

Efficiency of spat collection is affected by currents, depth, biological and chemical changes in water parameters, and hydrodynamics as well as the collector material types (Acarli et al., 2011; Coeroli et al., 1983; Monteforte and Garcia-Gasca, 1994; Knuckey, 1995; Friedman et al., 1998). Deployment timing of collector is critical for spat acquisition because bad timing triggers settlement of non-target species (Lok et al., 2006). Wild spat procurement from marine environment must be continuous and reliable for a sustainable bivalve aquaculture; in addition, success of this process depends on survival of new settled spat (Burke et al. 2008). The easily accessible and enduring settlement media (mesh bags) used for spat collection has proven effective for collection of many pearl oyster species (Beer and Southgate, 2006).

There has not been commercial enterprise for pearl culture in our country yet. Abundance of the pearl oyster spat on two different collector types, efficiency of collector materials, and the effect of environmental conditions on spat collecting were examined in this research. This study aims to determine the effect of collector types and water conditions on spat collection. It was also aimed to find out the best timing for spat attachment. This research is a fundemantally important work and considered leading the further studies about pearl culture.

\section{MATERIALS AND METHODS}

Spat collectors were deployed on a long line system in Karantina Island $\left(38^{\circ} 22^{\prime \prime} 44 \mathrm{~N}, 26^{\circ} 47^{\prime \prime} 12 \mathrm{E}\right)$ in the west of Izmir Bay between May, 2003 and June, 2004 (Figure 1). The water depth was nearly 10 meter with muddy and sandy sediment.

During the study, seawater parameters such as temperature, salinity, total particulate matter (TPM), and chlorophyll-a were followed. A mercury-in-glass thermometer with a range of -10 to $100 \pm 0.5{ }^{\circ} \mathrm{C}$ was used to measure the monthly temperature and a hand refractometer was used for salinity (ppt) measurements. TPM and chlorophyll-a were determined by filtering with GF/C filters (Strickland and Persons, 1972).

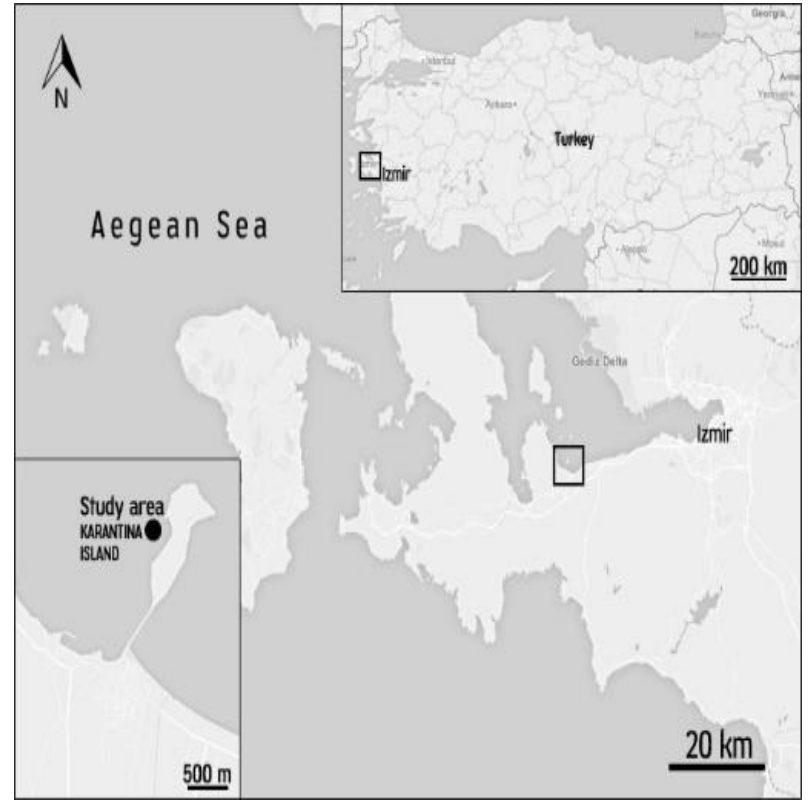

Figure 1. Study area at Urla Karantina Island

Two different models of collector were perapered for the study. Bag collectors which were originally used as "polyethylene bags" (size: $26 \times 35 \mathrm{~cm}, 0.91 \mathrm{~m}^{2}$, mesh size: $3 \times 3$ $\mathrm{mm}$ ) were put inside the polyethylene bodies for protection (Fig.2a). Shell collectors were consisted of mussel shells. For this purpose, fifteen mussel shells (average mussel shell size: $60.27 \pm 7.26 \mathrm{~mm}$ ) were fixed into a polyethylene bag $(26 \times 35 \mathrm{~cm})$ in a regular basis for each. Both collector types as one collector group were deployed on a long line at 1 meter below the surface in triplicate (Fig. 2b).

Six collector groups were installed in the following dates: group $(G) 1$ on May $23^{\text {rd }}, G 2$ on June $25^{\text {th }}$, G3 on July $21^{\text {st }}, \mathrm{G} 4$ on August 29th, $\mathrm{G} 5$ on September 29th , and G6 on November $07^{\text {th }}$. Three samples of each collector were removed from groups to observe the collection efficiency of pearl oyster spat at every deployment month. Spat shell length was measured from dorso-ventral axis. Fouling organisms (barnacles, polychaeta, bryozoa) and predators (crabs, gastropods) for Pinctada was recorded within the collectors. A scale was created for the counted fouling and predator species: 0-none; 1- few (1-19); 2-a few (20-39); 3- some (40-59); 4-many (6079); 5-most (80-99).

Obtained data for each group of collectors were analyzed by using Mann Whitney $U$ test. Monthly differences of recruitment were evaluated with Kruskal-Wallis. Statistical tests were performed using SPSS 15 software. Differences were considered significant when $p<0.05$. 


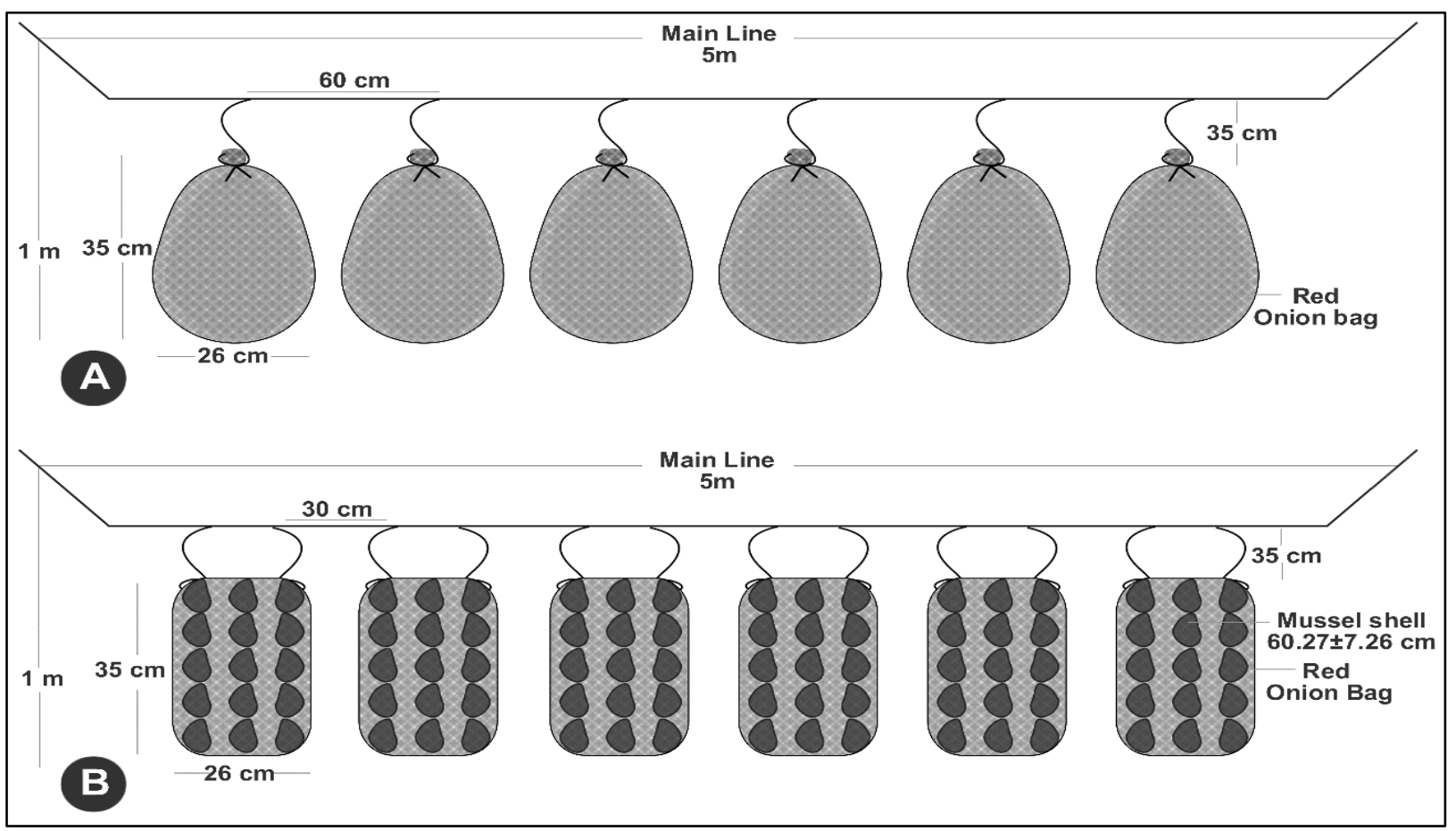

Figure 2. -(A) Bag and (B) shell collector design

\section{RESULTS}

During the study, temperature ranged between $12.5^{\circ} \mathrm{C}$ and $27^{\circ} \mathrm{C}$ in the seawater. The highest was measured in August, 2003 while the lowest was recorded in December, 2003. Average salinity was $\% 36.21 \pm 0.15$ (Fig. 3).

The highest TPM and chlorophyll-a were recorded as 33 $\mathrm{mg} / \mathrm{l}$ in August and $6.2 \mu \mathrm{g} / \mathrm{l}$ in September while the lowest values were observed $13 \mathrm{mg} / \mathrm{l}$ in July and $0.7 \mu \mathrm{g} / \mathrm{l}$ in January, respectively (Fig. 3b). From June to November, these data varied similarly and later on, TPM increased while chlorophylla decreased until January.

A total of $172 \pm 34.39 P$. radiata spat were obtained from both collector types during the study. Bag type collected $69.18 \%$ of pearl oyster spat while shell collectors did $30.82 \%$.

Significant difference was found between collector types $(p<0.05)$. Length range of spat on bag collectors was 4.47-33.9 $\mathrm{mm}$ and $4.66-18 \mathrm{~mm}$ on shell collectors. For bag collectors in $\mathrm{G} 2, \mathrm{G} 3$, and $\mathrm{G} 4$, average spat lenghts were $15.69 \pm 7.29$, $20.49 \pm 11.42$ and $8.20 \pm 1.99 \mathrm{~mm}$ and they were $12.69 \pm 4.32$, $11.96 \pm 4.59$ and $9.08 \pm 4.54 \mathrm{~mm}$ for shell collectors respectively.

$P$. radiata spat were not detected on $\mathrm{G} 1, \mathrm{G} 5$, and $\mathrm{G} 6$. Collector bags which belongs to $\mathrm{G} 1$ got lost on September $29^{\text {th }}$. Collector bags of $\mathrm{G} 5$ and $\mathrm{G} 6$ were excessively damaged on June, 29th. Therefore, collector bags on G1, G5, and G6 couldn't be examined.
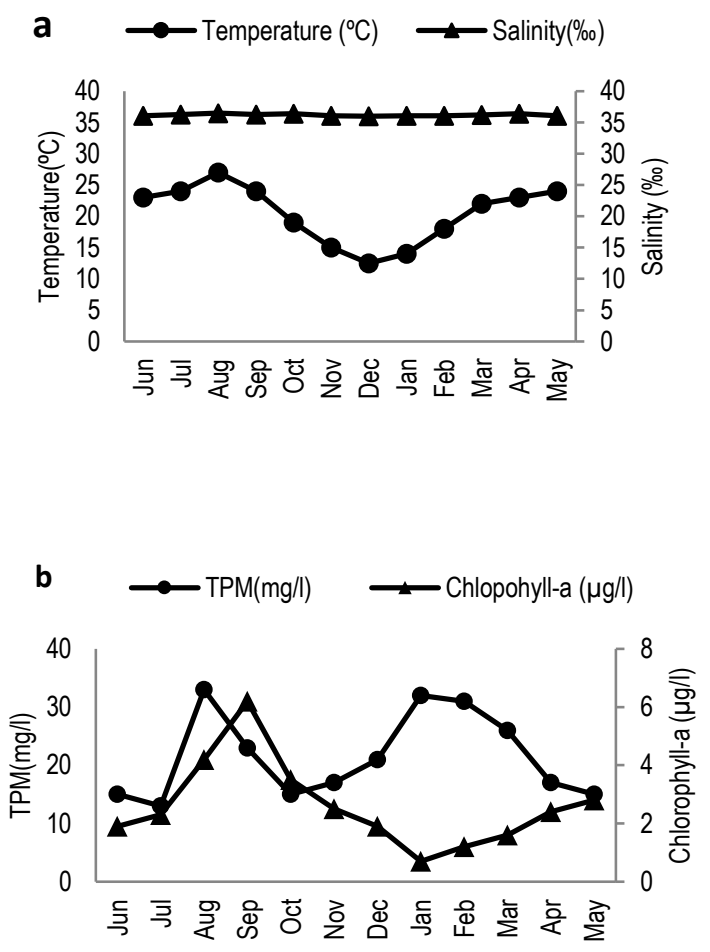

Figure 3. (a)Temperature $\left({ }^{\circ} \mathrm{C}\right)$ and salinity (\%o), (b) TPM and chlorophyll-a in study area 
First spat settlement on both collector types was observed at $\mathrm{G} 2$ on August, $29^{\text {th }}$. An abundance of $11.66 \pm 2.08$ individual (ind) $/ 0.91 \mathrm{~m}^{2}$ pearl oyster spat were collected from bag collectors while shell collectors accumulated $2.33 \pm 1.52$ ind./0.91 $\mathrm{m}^{2}$ in August (Table 1). Spat settlement on bag collectors was significantly higher than that of shell collectors $(p<0.05)$. It was defined that $100 \%$ of newly settled spat on G2 in August $29^{\text {th }}$ were smaller than $10 \mathrm{~mm}$ on bag collectors (Table 1). Finally, 15 ind./0.91 $\mathrm{m}^{2}$ were collected by bag collectors and 9 ind. $/ 0.91 \mathrm{~m}^{2}$ by shell collectors on $\mathrm{G} 2$.

More spat were collected with G3; 17 ind. $/ 0.91 \mathrm{~m}^{2}$ on bag and 13 ind. $/ 0.91 \mathrm{~m}^{2}$ on shell collector in September $29^{\text {th }}$. Total number of spat on $\mathrm{G} 3$ was determined to 29 ind./0.91 $\mathrm{m}^{2}$ on bag collectors and 28 ind./0.91 $\mathrm{m}^{2}$ on shell.

Whereas there were no $P$. radiata spat during first samplings in September 29th on G4, 34 ind. $/ 0.91 \mathrm{~m}^{2}$ and 22 ind./0.91 $\mathrm{m}^{2}$ were collected by bag and shell collectors respectively in the samplings of the other months. It was observed that spat within the collectors were continously growing with the extended underwater time. For example, in $\mathrm{G} 2$ on August $29^{\text {th }}, 100 \%$ of the spat were under $10 \mathrm{~mm}$ length, but in June $26^{\text {th }} 44.4 \%$ spat were over $20 \mathrm{~mm}$ length in the last sampling of same group.

Table 1. Mean number and size distrubution (\%) of settled pearl oyster $P$. radiata spat on bag and shell collector types

\begin{tabular}{|c|c|c|c|c|c|c|c|c|c|}
\hline & & \multicolumn{5}{|c|}{ Spat/Bag Collectors (\%) } & \multicolumn{3}{|c|}{ Spat/Shell Collectors (\%) } \\
\hline & Sampling Date & $\begin{array}{l}\text { Spat/Bag } \\
\text { collector }\end{array}$ & $<10 \mathrm{~mm}$ & $10-20 \mathrm{~mm}$ & $>20 \mathrm{~mm}$ & $\begin{array}{l}\text { Spat/Shell } \\
\text { collector }\end{array}$ & $<10 \mathrm{~mm}$ & $10-20 \mathrm{~mm}$ & $>20 \mathrm{~mm}$ \\
\hline \multirow{6}{*}{ 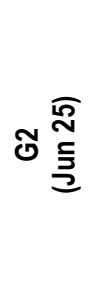 } & Jul 21 & - & - & - & - & - & - & - & - \\
\hline & Aug 29 & $11.66 \pm 2.08$ & 100.00 & - & - & $2.33 \pm 1.52$ & 50.00 & 50.00 & - \\
\hline & Sep 29 & $5.33 \pm 1.52$ & - & 40.00 & 60.00 & $2.33 \pm 1.52$ & - & 100.00 & - \\
\hline & Nov 07 & $12.33 \pm 1.52$ & 41.66 & 33.33 & 25.00 & - & - & - & - \\
\hline & Dec 29 & $13.66 \pm 2.08$ & 7.69 & 30.76 & 61.53 & $1.66 \pm 1.15$ & - & 100.00 & - \\
\hline & Jan 26 & $9.33 \pm 1.52$ & 11.11 & 44.44 & 44.44 & $4.33 \pm 1.52$ & 50.00 & 25.00 & 25.00 \\
\hline \multirow{6}{*}{ 刃ำ } & Aug 29 & $2.33 \pm 1.52$ & 100.00 & - & - & $4.33 \pm 1.52$ & 100.00 & - & - \\
\hline & Sep 29 & $17.33 \pm 1.52$ & 64.70 & 29.41 & 5.88 & $13.33 \pm 1.52$ & 61.53 & 30.76 & 7.69 \\
\hline & Nov 07 & $3.33 \pm 1.52$ & 33.33 & - & 66.66 & - & - & - & - \\
\hline & Dec 29 & $2.33 \pm 1.52$ & - & - & 100.00 & $1.66 \pm 1.15$ & - & 100.00 & - \\
\hline & Jan 26 & $3.33 \pm 1.52$ & - & - & 100.00 & $5.33 \pm 1.52$ & 20.00 & 40.00 & 40.00 \\
\hline & Mar 02 & $2.33 \pm 1.52$ & - & - & 100.00 & $5.33 \pm 1.52$ & 20.00 & 40.00 & 40.00 \\
\hline \multirow{6}{*}{ 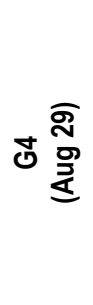 } & Sep 29 & - & - & - & - & - & - & - & - \\
\hline & Nov 07 & $9.33 \pm 1.52$ & 100.00 & - & - & - & - & - & - \\
\hline & Dec 29 & $9.33 \pm 1.52$ & 77.77 & 22.22 & - & $7.33 \pm 1.52$ & 57.14 & 42.85 & \\
\hline & Jan 26 & $8.33 \pm 1.52$ & 87.50 & 12.50 & - & $3.33 \pm 1.52$ & 100.00 & - & - \\
\hline & Mar 02 & $2.33 \pm 1.52$ & 100.00 & - & - & $12.33 \pm 1.52$ & 8.33 & 91.66 & \\
\hline & May 14 & $6.33 \pm 1.52$ & 50.00 & 50.00 & - & - & - & - & - \\
\hline
\end{tabular}

During the study 1147 macro organisms were observed on bag collectors except fouling and predators, and 2008 organisms were found on shell collectors. Some important species in the collectors are shown in Table 2.

Total number of collected bivalve species were 1022 and 1109 for bag and shell collectors, respectively. The larvae of predators also settled to spat collectors and grew rapidly so that large numbers of predators including crabs (Pilimnus hirtellus Linnaeus 1761, Psidia Iongimana Risso 1816) and Cymatium gastropods were found on the collectors (Table 3). The number of crab and cymatium gastropod was considerably higher on the bag collectors. It was found that fouling organisms (barnacles, polychaete, bryozoa) settled on shell collector in high numbers throughout the study. Bag collectors held less fouling organisms than shell collectors used during this study. Especially abundance of barnacle individuals on G1 and G4 shell collectors were more dominant. Almost every month barnacles and polychaets were observed on both type of collectors. 
Table 2. Abundance of species on bag and shell collectors (1. Pinctada radiata, 2. Ostrea edulis, 3. Mytilus galloprovincialis, 4. Chlamys glabra, 5. Chlamys varia, 6. Pinna nobilis, 7. Modiolus barbatus, 8. Cardium tuberculatum, 9. Arca noea, 10. Lima lima, 11. Anomia ephippium, 12. Paracentrotus lividus)(L-Lost/D-Damaged/B-Bag/S-Shell)

\begin{tabular}{|c|c|c|c|c|c|c|c|c|c|c|c|c|c|c|c|c|c|c|c|c|c|c|c|c|}
\hline Groups & Abu & ndan & ce of & Spec & es in & Bag & and $S$ & hell & ;ollec & ors & & & & & & & & & & & & & & \\
\hline G1 & 1B & 15 & $2 B$ & $2 S$ & 3B & $3 S$ & 4B & $4 S$ & $5 B$ & $5 S$ & 6B & $6 S$ & $7 B$ & $7 S$ & $8 B$ & $8 S$ & $9 \mathrm{~B}$ & 9S & $10 \mathrm{~B}$ & $10 S$ & 11B & $11 S$ & $12 B$ & $12 S$ \\
\hline Jun 25 & - & - & - & 4 & - & 4 & 11 & 5 & - & - & - & - & - & - & - & - & - & - & - & - & - & 8 & - & - \\
\hline Jul 21 & - & - & - & 10 & 1 & 10 & 23 & 1 & - & - & - & - & - & - & - & - & 18 & - & - & - & - & 18 & - & - \\
\hline Aug 29 & - & - & - & 13 & - & 13 & - & 1 & - & - & - & - & - & - & - & - & - & - & - & - & - & 18 & - & - \\
\hline Sep 29 & $\mathrm{~L}$ & $L$ & $\mathrm{~L}$ & $\mathrm{~L}$ & $\mathrm{~L}$ & $\mathrm{~L}$ & $\mathrm{~L}$ & $\mathrm{~L}$ & $L$ & $\mathrm{~L}$ & $\mathrm{~L}$ & L & $\mathrm{L}$ & L & $\mathrm{L}$ & $L$ & $L$ & $\mathrm{~L}$ & $\mathrm{~L}$ & $\mathrm{~L}$ & $\mathrm{~L}$ & $L$ & $L$ & $L$ \\
\hline Nov 07 & $\mathrm{~L}$ & L & $\mathrm{L}$ & L & $\mathrm{L}$ & $\mathrm{L}$ & $\mathrm{L}$ & $\mathrm{L}$ & $\mathrm{L}$ & $\mathrm{L}$ & $\mathrm{L}$ & L & L & $\mathrm{L}$ & L & L & L & L & $\mathrm{L}$ & $\mathrm{L}$ & $\mathrm{L}$ & $\mathrm{L}$ & $\mathrm{L}$ & $\mathrm{L}$ \\
\hline Dec 29 & $\mathrm{~L}$ & $\mathrm{~L}$ & $\mathrm{~L}$ & $\mathrm{~L}$ & $\mathrm{~L}$ & $L$ & $\mathrm{~L}$ & $\mathrm{~L}$ & $\mathrm{~L}$ & $L$ & $\mathrm{~L}$ & $L$ & $L$ & $L$ & $L$ & $L$ & $L$ & $\mathrm{~L}$ & $L$ & $\mathrm{~L}$ & $\mathrm{~L}$ & $L$ & $\mathrm{~L}$ & $\mathrm{~L}$ \\
\hline
\end{tabular}

G2

\begin{tabular}{|c|c|c|c|c|c|c|c|c|c|c|c|c|c|c|c|c|c|c|c|c|c|c|c|c|}
\hline Jul 21 & - & - & - & 21 & - & 21 & - & - & - & - & - & - & - & - & - & - & - & - & - & - & - & 4 & - & - \\
\hline Aug 29 & 11 & 2 & - & 91 & - & 91 & - & 3 & - & - & - & 1 & - & - & - & - & - & - & - & - & - & 24 & - & - \\
\hline Sep 29 & 5 & 2 & - & 74 & - & 74 & 3 & - & - & - & - & - & - & - & - & - & 20 & - & 16 & - & - & 20 & - & - \\
\hline Nov 07 & 12 & - & - & 34 & - & 34 & 7 & 2 & - & - & 2 & - & - & 3 & - & - & 1 & 6 & 2 & - & - & 11 & - & - \\
\hline Dec 29 & 13 & 1 & - & 25 & - & 25 & 6 & - & - & - & - & - & 2 & 12 & - & - & - & 1 & 4 & - & - & 21 & - & - \\
\hline Jan 26 & 9 & 4 & - & 62 & - & 62 & 2 & - & - & 2 & 1 & - & 1 & 9 & - & 1 & 1 & 6 & 1 & - & - & 38 & 2 & 11 \\
\hline
\end{tabular}

G3

\begin{tabular}{|c|c|c|c|c|c|c|c|c|c|c|c|c|c|c|c|c|c|c|c|c|c|c|c|c|}
\hline Aug 29 & 2 & 4 & - & 15 & - & 15 & 4 & 2 & - & - & - & - & - & - & - & - & - & - & - & - & - & 7 & - & - \\
\hline Sep 29 & 17 & 13 & - & 28 & 2 & 28 & 23 & 1 & - & - & 11 & - & - & - & - & - & - & - & 3 & - & - & 19 & - & - \\
\hline Nov 07 & 3 & - & 3 & 11 & - & 11 & 4 & - & - & - & 1 & - & 2 & 1 & - & - & 33 & - & 5 & - & - & 18 & - & - \\
\hline Dec 29 & 2 & 1 & - & 15 & 1 & 15 & - & - & - & - & - & - & 8 & - & 2 & - & 21 & - & 3 & - & - & 22 & - & - \\
\hline Jan 26 & 3 & 5 & - & 13 & 2 & 13 & 1 & - & - & 1 & - & 1 & 4 & - & - & - & 34 & 1 & 2 & - & - & 19 & 1 & 5 \\
\hline Mar 02 & 2 & 5 & 1 & - & 1 & - & - & - & - & 1 & - & - & 5 & 2 & - & - & 45 & 2 & 5 & - & - & - & - & 1 \\
\hline
\end{tabular}

G4

\begin{tabular}{|c|c|c|c|c|c|c|c|c|c|c|c|c|c|c|c|c|c|c|c|c|c|c|c|c|}
\hline Sep 29 & - & - & - & 1 & - & 1 & 1 & - & - & - & - & - & - & - & - & - & - & - & - & - & - & 12 & - & - \\
\hline Nov 07 & 9 & - & - & 7 & - & 7 & 37 & - & - & - & - & - & - & - & - & - & 8 & - & 5 & - & - & 6 & - & - \\
\hline Dec 29 & 9 & 7 & - & 15 & 1 & 15 & 26 & - & - & 1 & - & - & - & - & - & & 4 & 3 & 5 & - & - & 32 & 2 & - \\
\hline Jan 26 & 8 & 3 & - & 8 & - & 8 & 52 & 1 & - & 2 & 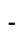 & - & 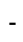 & - & 1 & 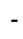 & 2 & 6 & 27 & - & - & 55 & 9 & 1 \\
\hline Mar 02 & 2 & 12 & - & - & - & - & 21 & - & - & 1 & - & 1 & - & 1 & - & - & 16 & 4 & 33 & - & - & - & - & 1 \\
\hline May 14 & 6 & - & - & 1 & - & 1 & 33 & 1 & - & - & - & - & - & - & - & - & 8 & - & 53 & - & - & - & - & - \\
\hline
\end{tabular}

G5

\begin{tabular}{|c|c|c|c|c|c|c|c|c|c|c|c|c|c|c|c|c|c|c|c|c|c|c|c|c|}
\hline Nov 07 & - & - & - & 4 & - & 4 & 9 & - & - & - & - & - & - & - & - & - & - & - & - & - & - & 96 & - & - \\
\hline Dec 29 & - & - & - & - & - & - & 5 & 1 & 1 & - & - & - & - & - & - & - & - & - & - & - & 1 & 61 & - & - \\
\hline Jan 26 & - & - & - & - & - & - & 30 & - & 1 & - & - & - & - & - & - & - & 3 & - & 4 & - & 1 & 83 & 4 & - \\
\hline Mar 02 & - & - & - & 1 & - & 1 & 9 & - & 2 & - & - & - & 1 & - & - & - & - & - & 14 & - & 2 & 79 & 11 & - \\
\hline May 14 & - & - & - & - & - & - & - & - & - & - & - & - & - & - & - & - & - & - & 6 & - & - & 51 & 4 & - \\
\hline Jun 29 & $D$ & $D^{*}$ & $D$ & $D$ & $D$ & $D$ & $D$ & $D$ & $D$ & $D$ & $D$ & $D$ & $D$ & $D$ & $D$ & $D$ & $D$ & $D$ & $D$ & $D$ & $D$ & $D$ & $D$ & $D$ \\
\hline \multicolumn{25}{|l|}{ G6 } \\
\hline Dec 29 & - & - & - & - & - & - & - & 5 & - & - & - & - & - & - & - & - & - & - & - & - & - & 25 & - & - \\
\hline Jan 26 & - & - & - & 13 & 8 & 13 & 17 & 2 & 33 & 10 & - & - & - & - & - & - & - & - & - & - & 33 & 54 & - & - \\
\hline Mar 02 & - & - & - & - & - & - & 8 & - & 48 & - & - & - & - & - & - & - & 2 & - & - & - & 48 & 79 & - & - \\
\hline May 14 & - & - & - & - & - & - & 1 & - & 20 & - & - & - & - & - & - & - & - & - & - & - & 20 & - & - & - \\
\hline Jun 29 & $D$ & $D$ & $D$ & $D$ & $D$ & $D$ & $D$ & $D$ & $D$ & $D$ & $D$ & $D$ & $D$ & $D$ & $D$ & $D$ & $D$ & $D$ & $D$ & $D$ & $D$ & $D$ & $D$ & $D$ \\
\hline
\end{tabular}


Table 3. Density of fouling organism on bag and shell collectors. ( $A$ Barnacles, B. Polychaete, C. Bryozoa, D.Crabs, E. Cymatium) [1-few (1-19), 2-a few(20-39), 3-some(40-59), 4- many(60-79), 5- most(8099)] (L-Lost/D-Damaged)

\begin{tabular}{|c|c|c|c|c|c|c|c|c|c|c|c|}
\hline \multirow{2}{*}{ Groups } & \multirow{2}{*}{$\begin{array}{l}\text { Sampling } \\
\text { date }\end{array}$} & \multicolumn{5}{|c|}{ Bag Collector } & \multicolumn{5}{|c|}{ Shell Collector } \\
\hline & & A & B & C & D & E & A & B & C & D & E \\
\hline \multirow[b]{6}{*}{$\overline{0}$} & Jun 25 & 1 & 1 & 1 & 0 & 0 & 4 & 1 & 1 & 0 & 0 \\
\hline & Jul 21 & 1 & 1 & 1 & 0 & 0 & 2 & 3 & 1 & 0 & 1 \\
\hline & Aug 29 & 1 & 1 & 1 & 1 & 1 & 2 & 3 & 1 & 1 & 1 \\
\hline & Sep 29 & L & L & $\mathrm{L}$ & $L$ & $\mathrm{~L}$ & $\mathrm{~L}$ & $\mathrm{~L}$ & $\mathrm{~L}$ & L & L \\
\hline & Nov 07 & L & L & L & L & $\mathrm{L}$ & $\mathrm{L}$ & L & $\mathrm{L}$ & $\mathrm{L}$ & L \\
\hline & Dec 29 & $\mathrm{~L}$ & $\mathrm{~L}$ & $\mathrm{~L}$ & $\mathrm{~L}$ & $\mathrm{~L}$ & $\mathrm{~L}$ & $\mathrm{~L}$ & $\mathrm{~L}$ & $\mathrm{~L}$ & $\mathrm{~L}$ \\
\hline \multirow{11}{*}{ ণิ } & Jul 21 & 1 & 1 & 1 & 0 & 0 & 2 & 1 & 2 & 0 & 0 \\
\hline & Aug 29 & 1 & 1 & 1 & 0 & 0 & 1 & 3 & 1 & 0 & 0 \\
\hline & Sep 29 & 1 & 1 & 1 & 1 & 0 & 2 & 5 & 1 & 0 & 1 \\
\hline & Nov 07 & 1 & 1 & 1 & 1 & 1 & 1 & 3 & 1 & 0 & 1 \\
\hline & Dec 29 & 1 & 1 & 1 & 1 & 1 & 2 & 2 & 1 & 1 & 1 \\
\hline & Jan 26 & 1 & 1 & 1 & 1 & 1 & 3 & 4 & 1 & 1 & 1 \\
\hline & Aug 29 & 1 & 1 & 0 & 0 & 0 & 1 & 2 & 0 & 0 & 0 \\
\hline & Sep 29 & 1 & 1 & 1 & 0 & 0 & 2 & 3 & 1 & 0 & 1 \\
\hline & Nov 07 & 1 & 1 & 1 & 1 & 1 & 2 & 3 & 1 & 1 & 1 \\
\hline & Dec 29 & 1 & 1 & 0 & 1 & 1 & 2 & 2 & 0 & 1 & 1 \\
\hline & Jan 26 & 1 & 1 & 1 & 1 & 1 & 2 & 4 & 1 & 1 & 1 \\
\hline \multirow[t]{6}{*}{ O } & Mar 02 & 1 & 1 & 1 & 1 & 1 & 2 & 3 & 1 & 1 & 1 \\
\hline & Sep 29 & 1 & 1 & 0 & 0 & 0 & 5 & 1 & 0 & 0 & 1 \\
\hline & Nov 07 & 1 & 1 & 0 & 0 & 0 & 4 & 1 & 1 & 0 & 0 \\
\hline & Dec 29 & 1 & 1 & 1 & 0 & 0 & 4 & 2 & 1 & 0 & 0 \\
\hline & Jan 26 & 1 & 1 & 0 & 1 & 1 & 4 & 1 & 0 & 0 & 0 \\
\hline & Mar 02 & 1 & 1 & 1 & 1 & 1 & 3 & 2 & 1 & 1 & 1 \\
\hline \multirow[t]{6}{*}{ 志 } & May 14 & 1 & 1 & 0 & 1 & 1 & 2 & 1 & 0 & 1 & 1 \\
\hline & Nov 07 & 1 & 1 & 1 & 0 & 0 & 1 & 2 & 1 & 0 & 0 \\
\hline & $\operatorname{Dec} 29$ & 1 & 1 & 1 & 1 & 1 & 1 & 2 & 1 & 0 & 0 \\
\hline & Jan 26 & 1 & 1 & 1 & 1 & 1 & 1 & 2 & 1 & 0 & 0 \\
\hline & Mar 02 & 1 & 1 & 1 & 1 & 1 & 3 & 1 & 1 & 1 & 0 \\
\hline & May 14 & 1 & 1 & 1 & 1 & 1 & 2 & 1 & 1 & 1 & 1 \\
\hline \multirow[t]{5}{*}{ 飐 } & Jun 29 & D & D & D & D & D & D & D & D & D & D \\
\hline & Dec 29 & 1 & 1 & 0 & 0 & 0 & 1 & 2 & 0 & 0 & 0 \\
\hline & Jan 26 & 1 & 1 & 1 & 0 & 0 & 1 & 2 & 1 & 0 & 0 \\
\hline & Mar 02 & 1 & 1 & 1 & 1 & 1 & 1 & 1 & 1 & 1 & 1 \\
\hline & May 14 & 1 & 1 & 1 & 1 & 1 & 1 & 2 & 1 & 1 & 0 \\
\hline 8 & Jun 29 & D & D & D & D & D & D & D & D & D & D \\
\hline
\end{tabular}

\section{DISCUSSION}

The collectors should be deployed a few weeks before spawning of target species; thus, primary bio-film layer occurs on the collector materials (Su et al., 2007). The collector systems that are deployed much earlier than regenerative phase will collect none target species and fouling life forms which compete with the target species (Yigitkurt, 2011). If collector systems are deployed after estimated larval settlement time, the spat collection capability of collector decreases and that can cause infrequency of pearl oyster spat (Ruzzante and Zaixso, 1985). Pearl oyster spat could not be found on both collector types in G1,G4, and G5. Absence of spat in $\mathrm{G} 1$ is relevant with too early deployment corresponding to pre-reproductive activity and G4 and G5 is considered to be outdated of the breeding season.

Temperature is a critical ecological agent in the regulation of oyster reproduction (Sastry, 1979). Spawning of bivalves is usually triggered by variation in sea water, such as increase and decrease in temperature or saltiness (Gervis and Sims, 1992). Pearl oyster spat were first observed in August, 2003, both on bag and shell collectors when the sea water temperature and salinity were the highest during the study. It is estimated that first reproductive activity began in late July or early August according to duration of metamorphosis. Reproductive activity was observed in a long period of the year, especially in July and August, considering newly settled individuals under $10 \mathrm{~mm}$ length which were determined on collectors. According to many studies, reproduction occurs by an increase in water temperature (Behzadi et al., 1997, Saucedo and Monteforte, 1997). Researchers noted that there is a positive corelation between recruited spat numbers and water temperature (Robinson et al., 1991) while studies revealed that there is no relationship between temperature and spat numbers (Harvey et al., 1995). No correlation was detected between temperature and spat collection in our study.

Environmental parameters, like chlorophyll-a and TPM, affect the growth and settlement of bivalves (MacDonald and Thompson, 1985; Gervis and Sims, 1992), which varies with site and depth (Lodeiros et al., 1998) of the water. In addition Wada, et al., (1995) reported that elements could affect regenerative cycle such as food availability and particulate matter. The largest number of pearl oyster spat were found on G3 in September which had the highest chlorophyll-a values. It was found that number of settled spat decreased by months when chlorophyll-a values were lower. For a successful oyster culture and pearl production nutrient-reach areas should be determined (Tomaru et al., 2002). Study region is suggested to be an appropriate location considering chlorophyll-a and TPM values during the study.

An average of 7.26 ind. $/ \mathrm{m}^{2}$ P.radiata spat were detected on bag collectors while it was 3.23 ind. $/ \mathrm{m}^{2}$ on shell collectors. Urban (2000) obtained 83 ind./ $\mathrm{m}^{2}$ and Núñez, et al., (2006) found 67 ind. $/ \mathrm{m}^{2}$ for $P$. imbricata spat. Another study which is conducted in La Paz Bay in Mexico, 22 ind. $/ \mathrm{m}^{2}$ of $P$. mazatlantica spat were observed on collectors (GaytanMondragon et al., 1993). In comparision with the other studies, a number of pearl oyster spat were less in this study. In the study area it can be declared that there were very few mature pinctada individuals when the number of obtained spat were taken into consideration.

Predation is an important cause for mortality of pearl oyster spat (Govan, 1995; Friedman and Bell, 1996; Friedman et al., 1998). Various predator species for bivalves settle on collectors during the planktonic phase and then grow with the other species (Friedman et al., 1998). In this research, the larvae of predators settled to collectors and grew so reached up to large 
numbers including crabs and gastropods on the collectors. However, broken shells of bivalve such as fan mussel and clam, which is thought to be consumed by these predator species. Furthermore, no serious damage was observed on $P$. radiata spat. Fouling organisms (barnacles, polychaeta, bryozoa) were also represented with high numbers on the shell collectors throughout the study. Detection in large quantities of fouling species on shell collectors is more attiributable to low abundance of spat in the area than the collector material.

The bag collector was more effective than shell collector to collect pearl oyster spat in the present study. Shell collector, containing hard surface structures, was more successful to collect edible oyster (0. edulis) spat (Lok and Acarli, 2006) which is in accordance with the other similar research in the world. During a study about Pinna nobilis spat collection, 183

\section{REFERENCES}

Acarli, S., Lök, A., \& Acarli, D. (2011). Preliminary spat settlement of fan mussel Pinna nobilis Linnaeus 1758 on a mesh bag collector in Karantina Island (Eastern Aegean Sea, Turkey). Fresenius Environmental Bulletin, 20:2501-2507.

Alagarswami, K. (1991). Production of cultured pearls. Indian Council of Agricultural Research, New Delhi:The Central Electric Press

Al-saadi, A. (2013). Population structure and patterns of genetic variation in a pearl oyster (Pinctada radiata ) native to the Arabian Gulf (MSc Thesis). Queensland University of Technology Brisbane, Australia, 1-109.

Beer, A.C., \& Southgate, P.C. (2000). Collection of pearl oyster (Family Pteriidae) spat at Orpheus Island, Great Barrier Reef (Australia). Journal of Shellfish Research, 19:5-7.

Baqueiro, E., \& Castagna, M. (1988). Fishery and culture of selected bivalves in Mexico: past, present and future. Journal of Shellfish Research, (7):433-443.

Beer, A.C., \& Southgate, P.C. (2006). Spat collection, growth and meat yield of Pinna bicolor (Gmelin) in suspended culture in northern Australia Aquaculture, 258:424-429. doi: 10.1016/j.aquaculture.2006.04.014

Behzadi, S., Parivar, K., \& Roustaian, P. (1997). Gonadal cycle of pearl oyster, Pinctada fucata (Gould) in Northeast Persian Gulf, Iran. Journal of Shellfish Research 16:129-135.

Burke, K., Bataller, É., \& Miron, G. (2008). Spat collection of a non-native bivalve species (European Oyster, Ostrea edulis) off the Eastern Canadian Coast. Journal of Shellfish Research, 27:345-353.

Chellam, A., Victor, A.C.C., Dharmaraj, S., Velayudhan, T.S., \& Satyanaryana Rao, T.S. (1991). Pearl oyster farming and pearl culture. Central Marine Fisheries Research Institue, 8

Coeroli, M., De Gaillande, D., Landret, J.P., \& Aquacop. (1983). Recent innovations in cultivation of molluscs in French Polynesia. 1st International Biennal Conference on Warm Water Aquaculture, Crustacea, Brigham Young University, Hawai, Feb9-11/1983, 348-384

Friedman, K.J., \& Bell, J.D. (1996). Effect of different substrata and protective mesh bags on collection of spat of the pearl oysters, Pinctada margaritifera (Linnaeus, 1758) and Pinctada maculata (Gould, 1850). Journal of Shellfish Research, 15:535-541.

Friedman, K.J., Bell, J.D., \& Tiroba, G. (1998). Availability of wild spat of the blacklip pearl oyster, Pinctada margaritifera, from 'open' reef systems in Solomon Islands. Aquaculture, 167:283-299. doi: $10.1016 / S 0044-8486(98) 00286-5$

Gaytan-Mondragon, I., Caceres-Martinez, C., \& Tobias-Sanchez, M. (1993) Growth of the pearl oyster Pinctada mazatlanica and Pteria sterna in different culture structures at La Paz Bay, Baja California Sur, Mexico.
$P$. radiata spat were collected by mesh bag collectors at the same site in 2007-2008 (Acarlı et al., 2011). In this study 119 \pm 22.60 spat of $P$. radiata were collected although this study carried out four years before Acarlı's study. Yiğitkurt (2011) reported 2059 pearl oyster spat; 1401 from surface waters and 658 from deeper waters around Urla Karantina Island. Significant differences on spat numbers between the mentioned studies are very engrossing in studies that carried out close years.

This is the first study of spat collection with two different collector materials for $P$. radiata in Turkey. This research is a fundemantally important work for commercial enterprises and similar researches. Subjects like reproductive activity, growth, and pearl production are required to get information about the biology of this species.

Journal of the World Aquaculture Society, 24:541-546. doi: 10.1111/j.1749-7345.1993.tb00585.x

Gervis, M.H., \& Sims, N.A. (1992). The biology and culture of pearl oysters (Bivalvia: Pteriidae). ICLARM Studies and Reviews, Manila, Philippines, 21:1-56.

Govan, H. (1995). Cymatium muricinum and other ranellid gastropods: major predators of cultured tridacnid clams. ICLARM Technical report, Manila, Philippines, 49:136.

Göksu, M.Z.L., Çevik, F., \& Findik, Ö. (2002). Lethal chlorine concentrations for the pearl oyster Pinctada radiata (Leach, 1814). Turkish Journal of Zoolgy, 26: 157-160.

Harvey, M., Bourget, E., \& Ingram, R.G. (1995). Experimental evidence of passive accumulation of marine bivalve larvae on filamentous epibenthic structures. Limnology and Oceanography, 40:94-104. doi: 10.4319//0.1995.40.1.0094

Knuckey, I.A. (1995). Settlement of Pinctada maxima (Jameson) and other bivalves on artificial collectors in the Timor Sea, Northern Australia. Journal of Shellfish Research, 14:411-416.

Lodeiros, C., Pico, D., Prieto, A., Narvaez, N., \& Guerra, A. (2002). Growth and survival of the pearl oyster Pinctada imbricata (Röding 1758) in suspended and bottom culture in the Golfo de Cariaco, Venezuela. Aquaculture International, 10:327-338. doi:10.1023/A:1022451608405

Lodeiros, J., Rengel, J., Freites, L., Morales, F., \& Himmelman, J.H. (1998). Growth and survival of the tropical scallop Lyropecten (Nodipecten) nodosus maintained in suspended culture at three depths. Aquaculture, 165:41-50. doi: 10.1016/S0044-8486(98)00212-9

Lok, A., \& Acarli, S. (2006). Preliminary study of settlement of flat oyster spat (Ostrea edulis L.) on oyster and mussel shell collectors. The Israeli Journal of Aquaculture-Bamidgeh, 58:2006. doi: 10.1016/S0044-8486(98)00212-9

Lok, A., Acarli, S., Serdar, S., Kose, A., \& Goulletquer, P. (2006). Growth and survival rates of bearded horse mussel (Modiolus barbatus Linne, 1758) in Mersin Bay (Turkey). The Israeli of Aquaculture-Bamidgeh, 58:55-61.

MacDonald, B., \& Thompson, R. (1985). Influence of temperature and food availability on the ecological energetics of the giant scallop Placopecten magellanicus. I. Growth rates of shell and somatic tissue. Marine Ecology Progress Series, 25:279-294. doi: 10.1007/BF00024236

Monteforte, M., \& Garcia-Gasca, A. (1994). Spat collection studies on pearl oysters Pinctada mazatlanica and Pteria sterna ( Bivalvia, Pteriidae) in Bahia de La Paz, South Baja California, Mexico. Hydrobiologia 291:2134. doi: $10.1007 / \mathrm{BF} 00024236$ 
Nayar, K.N., Mahadevan, S., Ramadoss, K., \& Rajan, C.T. (1978) Experimental study of the settlement and collection of pearl oyster spat from Tuticorin area. Indian Journal of Fisheries, 25(1\&2):246-252.

Núñez, P., Lodeiros, C., Acosta, V., \& Castillo, I. (2006). Seed reception of bivalve mollusks on different artificial substrates at Turpialito Bay, Cariaco Gulf, Venezuela. Zootecnia Tropical 24:483-496.

Urban, H.J., (2000). Culture potential of the pearl oyster Pinctada imbricata from the Caribbean. II. Spat collection, and growth and mortality in culture systems. Aquaculture 189:375-388 doi: 10.1016/S0044-8486(00)00394-X

Rahma, I.H., \& Newkirk, G.F. (1987). Economics of tray culture of the motherof-pearl shell Pinctada margaritifera in the Red Sea, Sudan. Journal of World Aquaculture Society, 18:156-161. doi: 10.1111/j.1749-7345.1987.tb00434.x

Robinson, M.C.S., Martin, J.D., \& Chandler, R.A. (1991). Spatial patterns of spat settlement in the sea scallop, Placopecten magellanicus, compared to hydrograpic conditions in Passamaquoddy Bay, New Brunswick, Canada. Journal of Shellfish Research, 10:272-273.

Ruzzante, D.E., \& Zaixso, H.E. (1985). Settlement of Chlamys tehuelchus (D'Orb.) on artificial collectors. Seasonal changes in spat settlement. Marine Ecology Progress Series 26:195-197.

Sastry, A.N. (1979). Pelecypoda (excluding Ostreidae). In Giese A.C., Pearse J.S., (Ed.), Reproduction of marine invertebrates: Molluscs: Pelecypods and lesser classes (pp 131-195), New York: Academic Press. doi: 10.1016/B978-0-12-282505-7.50012-9
Saucedo, P., \& Monteforte, M. (1997). Breeding cycle of pearl oysters Pinctada mazatlanica and Pteria sterna (Bivalvia: Pteriidae) at Bahia de La Paz Baja California Sur, Mexico. Journal of Shellfish Research, 16:103-110.

Strickland, J.D.H., \& Parsons, T.R. (1972). A practical handbook of seawater analysis. Fisheries Research Board of Canada, Bulletin, 167.

Su, Z., Huang, L., Yan, Y., \& Li, H. (2007). The effect of different substrates on pearl oyster Pinctada martensii (Dunker) larvae settlement. Aquaculture, 271:377-383. doi: 10.1016/j.aquaculture.2007.02.039

Tomaru, Y., Kumatabara, Y., Kawabata, Z., \& Nakano, S. (2002). Effect of water temperature and chlorophyll abundance on shell growth of the Japanese pearl oyster, Pinctada fucata martensii, in suspended culture at different depths and sites. Aquaculture Research 33:109-116. doi: 10.1046/j.1365-2109.2002.00653.x

Vakily, J.M. (1989). The biology and culture of mussels of the genus Perna. ICLARM Studies and Reviews, Manila, Philippines, 17:1-56.

Victor, A.C.C., Chellam, A., \& Dharmaraj, S. (1987). Pearl oyster spat collection. Central Marine Fisheries Research Institue Bulletin, 39:49-53.

Wada, K.T., Komaru, A., Ichimura, Y., \& Kurosaki, H. (1995). Spawning peak occurs during winter in the Japanese subtropical population of the pearl oyster, Pinctada fucata fucata. Aquaculture, 133:207-214. doi: 10.1016/0044-8486(95)00011-P

Yigitkurt, S. (2011). Effects of biotic and abiotic factors to pearl oyster (Pinctada radiata Leach, 1814) culture. (PhD Thesis) Ege University, 141 